\title{
Uniaxial pressure in a High-resolution X-ray Diffraction environment
}

\author{
V. E. S. Frehse ${ }^{1,2}$, G. A. Lombardi ${ }^{1,2}$, J. C. Corsaletti Filho ${ }^{1}$, C. Adriano ${ }^{2}$, R. D. dos Reis ${ }^{1}$, G. A. Calligaris ${ }^{1}$ \\ ${ }^{1}$ Brazilian Synchrotron Light Laboratory, Brazilian Center for Research in Energy and Materials, Campinas, SP, 13083-970, Brazil, \\ ${ }_{2}^{2}$ Institute of Physics "Gleb Wataghin", University of Campinas - UNICAMP, 13083-859, Campinas, SP, Brazil
}

vinicius.frehse@lnls.br

Improvement to macroscopic features such as better conductivity, weather resistance, and improved capacity and speeds for digital storage is in high demand for the ever-growing electronic-device market. A key point in this development is to relate these features with the atomic and electronic structure of the material in interest, regardless of knowledge area (e.g. chemistry, physics, engineering). With this in mind, we intend to develop an infrastructure that supports both control and analysis of such structural properties based on uniaxial pressure and advanced synchrotron-based techniques. For this, a compact cell [1] allows a consistent uniaxial strain to sub-millimetersized samples over a temperature range between 0.3 and $325 \mathrm{~K}$. Its clever design guarantees easy access to the sample, which also allows electrical contacts into it and provides a great solid angle for XRD experiments. In addition to it, the cell also supports magnetic fields up to $30 \mathrm{~T}$. On the other hand, a code based on the xrayutilities [2] python package allows an efficient description of the material in interest via its composition, crystalline structure and, most essentially in our case, its elastic properties. Therefore, it plays an essential role in tracking or predicting the sample's structural changes depending on its orientation and the uniaxial strain direction, as is shown in Fig. 1, where a uniaxial stress sweep was applied to sapphire $\left(\mathrm{Al}_{2} \mathrm{O}_{3}\right)$ to obtain its lattice parameters at each state.

Here, we exhibit the status of this uniaxial strain infrastructure present at the EMA beamline of Sirius [3], which can be used with other extreme thermodynamic conditions such as high magnetic field (up to 11T) and low temperatures (down to 0.5K). Particularly for XRD experiments, the uniaxial strain cell conveniently sits on top of a 6-circle $(4 \mathrm{~S}+2 \mathrm{D})$ diffractometer (Fig. 2) that supports conventional single-crystal experiments and high-resolution Reciprocal Space Mapping (RSM), which are great tools to probe strain and crystal symmetry breaking. This approach will open a plethora of opportunities for fine-tuning of properties of advanced materials, such as the Heusler material $\mathrm{Mn}_{3} \mathrm{Ge}$, also shown here. Given its various crystalline structures with different magnetic ordering (cubic $\rightarrow$ ferrimagnetic, tetragonal $\rightarrow$ ferromagnetic with momenta along the $\mathrm{c}$ axis, hexagonal $\rightarrow$ non-collinear antiferromagnetic with Anomalous Hall Effect), it is a particularly interesting material, and it being theoretically possible to change between each structure using pressure in specific directions [4] offer a great potential of exploration.

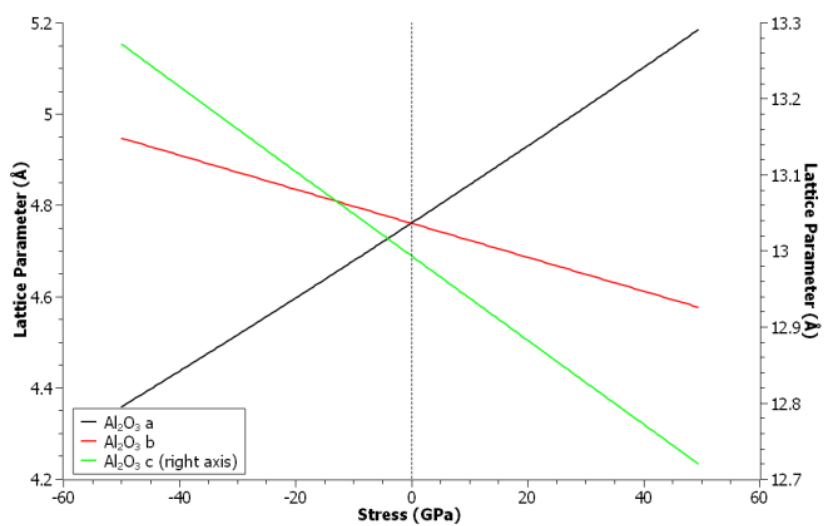

Figure 1. Strain $\mathrm{x}$ stress analysis of $\mathrm{Al}_{2} \mathrm{O}_{3}$ using our developed function

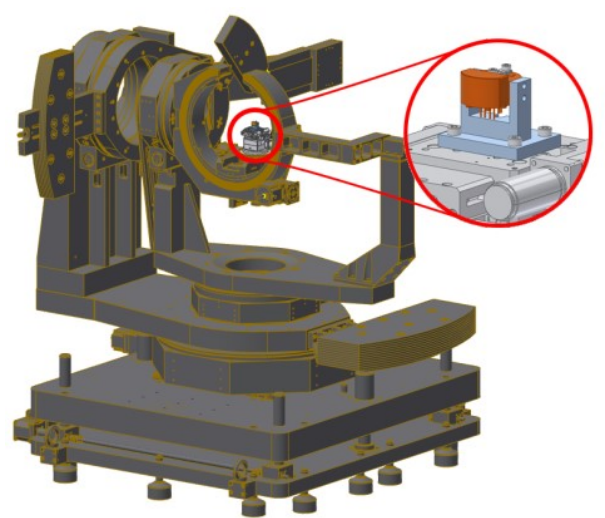

Figure 2. 6-circle diffractometer with the Strain Cell in our designed support on the inset

[1] Clifford W. Hicks, Mark E. Barber, Stephen D. Edkins, Daniel O. Brodsky, and Andrew P. Mackenzie, "Piezoelectric-based apparatus for strain tuning", Review of Scientific Instruments 85, 065003 (2014) https://doi.org/10.1063/1.4881611

[2] Kriegner, D., Wintersberger, E. \& Stangl, J. (2013). J. Appl. Cryst. 46, 1162-1170.

[3] R. D. dos Reis et al 2020 J. Phys.: Conf. Ser. 1609012015

[4] T. Graf, C. Felser, S. S. P. Parkin (2011). Simple rules for the understanding of Heusler compounds. Progress in Solid State Chemistry, Volume 39, Issue 1, Pages 1-50, ISSN 0079-6786. https://doi.org/10.1016/j.progsolidstchem.2011.02.001.

Keywords: Uniaxial pressure; Single crystal diffraction; Synchrotron beamline; $\mathrm{Mn}_{3} \mathrm{Ge}$ 